\title{
NEGLIGIBLE SETS FOR REAL CONNECTIVITY FUNCTIONS
}

\author{
JACK B. BROWN
}

Introduction. Only functions from the interval ${ }^{m} I=[0,1]$ into $I$ will be considered in this paper, and no distinction will be made between a function and its graph. For real functions $f$ from $I$ into $I$, the property of connectivity is intermediate to that of continuity and the Darboux property, and is equivalent to the property of $f$ being a connected set in the plane. For some connectivity functions $g$ from $I$ into $I$, it can be observed that there is a subset $M$ of $I$ such that every function from $I$ into $I$ which agrees with $g$ on $I-M$ is a connectivity function (in this case, $M$ will be said to be $g$-negligible). For example, if $M$ is a Cantor subset of $I$ and $g$ is a function from $I$ into $I$ such that if $x$ belongs to some component $(a, b)$ of $I-M, g(x)=\mid \sin \{\cot [\pi(x-a) /(b-a]\} \mid$, then $M$ is $g$-negligible. The following theorems will be proved:

THEOREM 1. If $M$ is a subset of $I$, then the following statements are equivalent:

(i) there is a connectivity function $g$ from $I$ into $I$ such that $M$ is g-negligible, and

(ii) every subinterval of $I$ contains c-many points of $I-M$ ( $c$ denotes the cardinality of the continuum).

THEOREM 2. If $g$ is a connectivity function from I into $I$, then the following statements are equivalent:

(i) $g$ is dense in $I^{2}$,

(ii) every nowhere dense subset of $I$ is g-negligible, and

(iii) there exists a dense subset of $I$ which is g-negligible. "Nowhere dense" cannot be replaced with "countable" in (ii), but the following is true:

THEOREM 3. There exists a connectivity function $g$ from $I$ into $I$ such that every subset of $I$ which has cardinality less than $c$ is g-negligible.

It follows from Theorem 1 that there is a connectivity function $g$ from $I$ into $I$ such that there is a $g$-negligible set $M$ of length one. However, since well-ordering techniques are used in the proof of Theorem 1, it might be difficult to imagine what such a function is actually like. Therefore, an effective example will be given of a con-

Received by the editors February 7, 1969. 
nectivity function $g$ from $I$ into $I$ for which there is a $g$-negligible set of length one. In proving that this example is a connectivity function, a fairly general construction theorem will be established, which may prove useful in the future construction of pathological connectivity functions.

Proofs of theorems. Several of the proofs given will rely on the following lemma which is an immediate corollary to the theorem in [1]. A brief outline of a direct proof is given.

Lemma. Suppose $f$ is a function from I into I such that if $g$ is a lower semicontinuous function from $I$ into $I$ and $J$ is a subinterval of $I$, then $f$ and $g$ agree at some element of $J$. Then $f$ is a connectivity function.

Outline of Proof. Suppose $f$ is not a connectivity function. Then $f$ is the union of two mutually separated sets $h$ and $k$ and there are mutually exclusive open subsets $U$ and $V$ of $I^{2}$ which contain $h$ and $k$, respectively. The projections of $U$ and $V$ onto $I$ are open and their union is $I$, so these projections have an element $x$ in common. There is an interval $[p, q]$ which contains $x$ such that for some two numbers $a$ and $b$, the interval with endpoints $(p, b)$ and $(q, b)$ is a subset of $U$ and the interval with endpoints $(p, a)$ and $(q, a)$ is a subset of $V$. Assume that $a<b$. Then the function $g$ defined by $g(y)=\sup \{t \mid$ the interval with endpoints $(y, a)$ and $(y, t)$ is a subset of $V\}$ if $y$ is in $[p, q]$, and $g(y)=1$ otherwise, is lower semicontinuous, and $g \mid[p, q]$ is a subset of the boundary of $V$ and does not intersect $f$, contrary to hypothesis.

Proof of Theorem 1. Suppose $M$ is a subset of $I$ such that every subinterval of $I$ contains $c$-many points of $I-M$. There is a collection $W$ of mutually exclusive subsets of $I-M$ such that $W$ has cardinality $c$ and each set in $W$ is dense in $I$. Let $T$ be a reversible transformation from $W$ onto the collection $S$ of all lower semicontinuous functions from $I$ into $I$. Let $g$ be a function from $I$ into $I$ such that if $x$ belongs to some element $w$ of $W$ and $h=T(w)$, then $g(x)=h(x)$. Clearly, any function $f$ from $I$ into $I$ which agrees with $g$ over $I-M$ will have the property that if $h$ is a lower semicontinuous function from $I$ into $I$ and $J$ is a subinterval of $I$, then $f$ and $h$ agree at some element of $J$. Thus, $M$ is $g$-negligible.

Now, suppose $M$ is a subset of $I,[a, b]$ is a subinterval of $I$ which does not contain $c$-many points of $I-M$, and $g$ is a function from $I$ into $I$. There is a number $t$ between 0 and 1 which is not in $\{g(x) \mid x$ is in $[a, b]-M\}$. Let $f$ be a function from $I$ into $I$ which agrees with $g$ over $I-M$ and has the property that if $x$ is in $M$, then $f(x)=t / 2$ if $x \leqq(a+b) / 2$ and $f(x)=(t+1) / 2$ if $x>(a+b) / 2$. Let $x$ and $y$ be ele- 
ments of $[a, b] \cap M$ such that $x<(a+b) / 2$ and $y>(a+b) / 2 . f$ is separated by the set which is the union of the interval from the point $(x, t)$ to $(y, t)$, the vertical ray extending upward from $(x, t)$, and the vertical ray extending downward from $(y, t)$. Therefore $M$ is not $g$-negligible.

Proof of Theorem 2. To show that (i) implies (ii), suppose $g$ is a connectivity function from $I$ into $I$ which is dense in $I^{2}, M$ is a nowhere dense subset of $I$, and $f$ is a disconnected function from $I$ into $I$ which agrees with $g$ over $I-M$. Assume $f=H \cup K$, where $H$ and $K$ are mutually separated subsets of $I^{2}$. Let $N=\mathrm{Cl}(M)$. The restriction $f \mid C$ of $f$ to any component $C$ of $I-N$ is $g \mid C$ and is therefore connected and lies entirely in $H$ or entirely in $K$. Suppose $C$ is a component of $I-N$ and that $f \mid C$ lies in $H$. Suppose there is a component $D$ of $I-N$ such that $f \mid D$ lies in $K$. Assume $D$ is to the right of $C$. The least upper bound of the set $\{x \mid x$ is greater than the left end $a$ of $C$, and if $a<y<x$ and $y$ belongs to a component $E$ of $I-N$, then $f \mid E$ lies in $H\}$ is a number $y$ such that either

(1) the point $(y, f(y))$ is in $K$ and $y$ is a limit number of the union of all components $E$ of $I-N$ such that $f \mid E$ lies in $H$ or

(2) the point $(y, f(y))$ is in $H$ and $y$ is a limit number of the union of all components $E$ of $I-N$ such that $f \mid E$ lies in $K$.

Notice that if, contrary to earlier supposition, there is no component $D$ of $I-N$ such that $f \mid D$ lies in $K$, then there will exist a number $y$ with property (1). Assume $y$ has property (1) and suppose $\epsilon>0$. Let $E$ be a component of $I-N$ such that $f \mid E$ lies in $H$ and $E$ has elements that lie within $\epsilon$ of $y$. Then the $\epsilon$-neighborhood $R$ of the point $(y, f(y))$ intersects the set $E \times I$, and since $f \mid E$ is dense in $E \times I$, $R$ contains a point of $f \mid E$. This means that $(y, f(y))$ is a limit point of $H$, which is a contradiction. A similar contradiction occurs if $y$ has property (2), so (i) implies (ii).

It is easily seen that (ii) implies (i) and (iii) implies (i), for suppose $R$ is a circular region lying in $I^{2}$ which contains no point of the connectivity function $g$ from $I$ into $I$, and suppose $M$ is a dense subset of $I$. There is an element $x$ of $M$ which is the abscissa of a point $(x, t)$ of $R$. The function $f$ from $I$ into $I$ which agrees with $g$ on $I-\{x\}$ and is such that $f(x)=t$ is not a connectivity function. Thus $M$ is not $g$-negligible, and $\{x\}$ is a nowhere dense subset of $I$ which is not $g$-negligible.

Now, suppose $g$ is a connectivity function from $I$ into $I$ which is dense in $I^{2}$. Let $H=\{x \mid g(x)=1 / 2\}$ and suppose there is a subinterval $J$ of $I$ which contains no element of $H$. There is an element $x$ of $J$ such that $g(x)>1 / 2$ and an element $y$ of $J$ such that $g(y)<1 / 2 . g$ is 
separated by the set which is the union of the interval from the point $(x, 1 / 2)$ to $(y, 1 / 2)$, the vertical ray extending downward from $(x, 1 / 2)$ and the vertical ray extending upward from $(y, 1 / 2)$. This is contradictory, so $H$ must be dense in $I$. Then $H=M \cup K$, where $M$ and $K$ are mutually exclusive and dense in $I$ and $M$ is countable. Suppose $f$ is a function from $I$ into $I$ which agrees with $g$ on $I-M$, and $f=A \cup B$ where $A$ and $B$ are mutually separated. Let $V$ and $W$ be mutually exclusive open sets such that $A$ is a subset of $V$ and $B$ is a subset of $W$. Suppose there is a region $R$ lying in $V \cap I^{2}$ which contains no points of $f$ with abscissa in $I-M$. Then since $M$ is countable, there will be numbers $x, y$, and $t$ such that $t \neq 1 / 2$, the interval from $(x, t)$ to $(y, t)$ lies in $R$ and contains no point of $f$ or $g$, and $g(x)>t$ and $g(y)<t$. $g$ would be disconnected by a set similar to those described before. Therefore $V$ and, for similar reasons, $W$ both contain points of $f$ with abscissa in $I-M$. Since $K$ is dense in $I$, the set $N=\{x \mid x$ is in $M$ and the point $(x, 1 / 2)$ is not in $V \cup W\}$ is nowhere dense. But the function $h$ from $I$ into $I$ which agrees with $f$ on $N$ and agrees with $g$ on $I-N$ lies in $V \cup W$, contains a point of $V$ and a point of $W$, and is therefore disconnected. This means that $N$ is a nowhere dense subset of $I$ which is not $g$-negligible. This is a contradiction, so (i) implies (iii).

The phrase "nowhere dense" cannot be replaced by the word "countable" in (ii) because the example described by J. L. Cornette on pp. 190 and 191 of [3] is a connectivity function $g$ from $I$ into $I$ which is dense in $I^{2}$, but the countable set $\left\{x \mid x=m / 2^{n}\right.$ for some positive integer $n$ and some nonnegative integer $\left.m \leqq 2^{n}\right\}$ is not $g$-negligible.

Proof of Theorem 3 . Let $F$ be the function defined in the proof of Theorem 3 of [2]. The function $f$ from $I$ into $I$ defined by $f(t)$ $=F(t)$ if $F(t)$ is in $I$, and $f(t)=0$ otherwise, will have the desired properties.

Description of the example. Let $M(1)$ be the middle third Cantor subset of $I$, and for each integer $n>1$, let $M(n)=\{x \mid x$ belongs to $M(n-1)$ or to the middle third Cantor subset of the closure of some component of $I-M(n-1)\}$. If $e$ is a number between 0 and 1 and $(c, d)$ is a segment, then the segment $(c+(d-c)(1-e) / 2, d-(d-c)$ $(1-e) / 2)$ shall be called the "middle $e$ th of $(c, d)$ ". Let $r_{1}, r_{2}, \cdots$ be a sequence of numbers in $I$ such that each element of $I$ is the sequential limit of some subsequence of $r_{1}, r_{2}, \ldots$ Let $f_{1}, f_{2}, \ldots$ be a sequence of functions from $I$ into $I$ with the following properties:

(i) $f_{1}$ is continuous on $I$, constant on each component of $I-M(1)$, and $f_{1}(x)=r_{1}$ if $x$ is in the middle $(1-1 / 2)$ th of $I$, and 
(ii) if $n$ is an integer greater than $1, f_{n}$ agrees with $f_{n-1}$ on $M(n-1)$, is constant on each component of $I-M(n)$, and if $C$ is a component of $I-M(n-1)$, then $f_{n}$ is continuous on $\mathrm{Cl}(C)$ and $f_{n}(x)=r_{n}$ if $x$ is in the middle $\left(1-1 / 2^{n}\right)$ th of $C$.

Let $M=\{x \mid$ there is a positive integer $n$ such that if $j$ is an integer greater than $\left.n, f_{j}(x)=r_{j}\right\} . M$ has length one because if for each positive integer $n, H_{n}=\left\{x \mid x\right.$ is not in the middle $\left(1-1 / 2^{n}\right)$ th of a component of $I-M(n)\}$, then

$$
K=\bigcap_{j=1}^{\infty} \bigcup_{i=j}^{\infty} H_{i}
$$

has length zero, and $I-K$ is a subset of $M$. Let $g$ be a function from $I$ into $I$ such that if $x$ is in $M, g(x)=0$, and if $x$ is in $I-M, g(x)$ is the sequential limit of some subsequence of $f_{1}(x), f_{2}(x), \cdots$. Since each element of $I$ is the sequential limit of some subsequence of $r_{1}, r_{2}, \cdots$, then every function $f$ from $I$ into $I$ which agrees with $g$ on $I-M$ would satisfy the hypothesis of the following theorem, and would therefore be a connectivity function.

Theorem 4. Suppose

(i) $M(1), M(2), \cdots$ is a monotonic increasing sequence of Cantor subsets of $I$ such that if $j$ is a positive integer and $C$ is a component of $I-M(j)$, then $\mathrm{Cl}(C) \cap M(j+1)$ is a Cantor set, and $M(1) \cup M(2) \cup \ldots$ is dense in $I$, and

(ii) $f_{1}, f_{2}, \ldots$ is a sequence of functions from $I$ into $I$ such that $f_{1}$ is continuous on $I$ and constant on each component of $I-M(1)$, and if $n$ is an integer, $n>1, f_{n}$ agrees with $f_{n-1}$ on $M(n-1)$, is constant on each component of $I-M(n)$, and is continuous on the closure of each component of $I-M(n-1)$.

Then if $f$ is a function from $I$ into $I$ such that for each $x$ in $I, f(x)$ is the sequential limit of some subsequence of $f_{1}(x), f_{2}(x), \cdots, f$ is a connectivity function.

Proof of Theorem 4. Notice that if $n$ is a positive integer, $f\left|M(n)=f_{n}\right| M(n)$, and if $\epsilon>0$, there is a positive integer $n$ such that the length of each component of $I-M(n)$ is less than $\epsilon$.

If $n$ is a positive integer, the statement that a point set $g$ is of type $n$ means that for each component $C=(a, b)$ of $I-M(n)$ there is a connected point set $A_{C}$ which contains $(a, f(a))$ and $(b, f(b))$, and $g=\left\{A_{C} \mid C \text { is a component of } I-M(n)\right\}^{*} \cup[f \mid M(n)]$ (if $G$ is a collection of sets, $G^{*}$ is the union of the sets in $G$ ). Suppose $g$ is a point set of type 1 , and $g=H \cup K$, where $H$ and $K$ are mutually separated. If $C$ and $D$ are components of $I-M(1)$, then $A_{C}$ lies entirely in $H$ or 
in $K$, and if $A_{C}$ and $A_{D}$ intersect, they both lie in $H$ or they both lie in $K$. Let $H^{\prime}$ be the set such that $P$ belongs to $H^{\prime}$ if and only if $P$ is in $H \cap[f \mid M(1)]$ or $P$ is a point of $f_{1}$ with abscissa in a component $C$ of $I-M(1)$ such that $A_{C}$ lies in $H$. Let $K^{\prime}=f_{1}-H^{\prime}$. Since $f_{1}$ is continuous, $H^{\prime}$ and $K^{\prime}$ are not mutually separated, so assume $P$ is a point of $H^{\prime}$ which is a limit point of $K^{\prime}$. $P$ must be a point of $f \mid M(1)$ and therefore in $H$. There is a neighborhood $R$ about $P$ which contains no point of $K . R$ contains a point $Q$ of $K^{\prime}$. If $Q$ has abscissa in a component $(a, b)$ of $I-M(1)$, then $(a, f(a))$ is a point of $K$ in $R$ or $(b, f(b))$ is a point of $K$ in $R$, and if $Q$ has abscissa in $M(1)$, then $Q$ is in $K$. Both of these possibilities are contradictions, so $g$ is connected.

Now, suppose $n$ is an integer greater than 1 and $g$ is a point set of type $n$. If $D$ is a component of $I-M(n-1)$, then the point set $B_{D}$ $=\left\{A_{C} \mid C\right.$ is a component of $\left.\mathrm{Cl}(D)-M(n)\right\} * \cup[f \mid \mathrm{Cl}(D) \cap M(n)]$ is connected for the same reasons sets of type 1 are connected, so $g$ is of type $n-1$. Therefore, it follows inductively that if $n$ is a positive integer and $g$ is of type $n$, then $g$ is connected.

Suppose $f$ is as described in the theorem, but $f=H \cup K$, where $H$ and $K$ are mutually separated. Let $V$ and $W$ be mutually exclusive open sets containing $H$ and $K$, respectively, and let $Q=I^{2}-(V \cup W)$. Let $M=M(1) \cup M(2) \cup \ldots$. Suppose $f \mid M$ lies entirely in $V$. Let $x$ be an element of $I-M$ such that $(x, f(x))$ is in $K$. For each positive integer $n$, let $\left(a_{n}, b_{n}\right)$ be the component of $I-M(n)$ which contains $x$. For each positive integer $n, f_{n}$ is constant on each component of $I-M(n)$, so the point $(x, f(x))$ is the sequential limit point of some subsequence of the point sequence $\left(a_{1}, f_{1}\left(a_{1}\right)\right),\left(a_{2}, f\left(a_{2}\right)\right), \cdots$, and this is a contradiction because the points in that sequence are in $H$. Therefore, $f \mid M$ contains a point of $H$ and a point of $K$, and there is a positive integer $n$ such that $f \mid M(n)$ does not lie entirely in one component of $V \cup W$. Suppose that for each component $C=(a, b)$ of $I-M(n)$, $(a, f(a))$, and $(b, f(b))$ belong to the same component $U$ of $V \cup W$, and $A_{C}$ is an arc with ends $(a, f(a))$ and $(b, f(b))$ which lies in $U$. Then, the set $\left\{A_{C} \mid C\right.$ is a component of $\left.I-M(n)\right\} * \cup[f \mid M(n)]$ would be a connected subset of $V \cup W$ which does not lie entirely in a single component of $V \cup W$, and this is a contradiction. Thus, there is a component $\left(a_{1}, b_{1}\right)$ of $I-M(n)$ such that $\left(a_{1}, f\left(a_{1}\right)\right)$ and $\left(b_{1}, f\left(b_{1}\right)\right)$ lie in different components of $V \cup W$ (the interval between them will intersect $Q)$. Thus, a sequence $\left(a_{1}, b_{1}\right),\left(a_{2}, b_{2}\right), \cdots$ can be defined so that if $j$ is an integer greater than $1,\left(a_{j}, b_{j}\right)$ is a component of $I-M(n+j-1)$ such that $a_{j-1}<a_{j}<b_{j}<b_{j-1}$, and $\left(a_{j}, f\left(a_{j}\right)\right)$ and $\left(b_{j}, f\left(b_{j}\right)\right)$ lie in different components of $V \cup W$ (the interval between them will intersect 
$Q)$. Let $x$ be the element common to the segments $\left(a_{1}, b_{1}\right),\left(a_{2}, b_{2}\right), \ldots$. $f(x)$ must be the sequential limit of some subsequence of $f_{1}(x)$, $f_{2}(x), \cdots$. But since for each positive in teger $n, f_{n}$ is constant on each component of $I-M(n), f(x)$ is also the sequential limit of some subsequence of $f\left(a_{1}\right), f\left(a_{2}\right), \cdots$. However, if $c_{1}, c_{2}, \ldots$ is a subsequence of $a_{1}, a_{2}, \cdots$ such that $f(x)$ is the sequential limit of $f\left(c_{1}\right), f\left(c_{2}\right), \cdots$, then the point $(x, f(x))$ is the sequential limit point of the point sequence $\left(c_{1}, f\left(c_{1}\right)\right),\left(c_{2}, f\left(c_{2}\right)\right), \cdots$, and this point sequence must converge to a point of $Q$. This is a contradiction, so the theorem is proved.

\section{REFERENCES}

1. J. B. Brown, Connectivity, semi-continuity, and the Darboux property, Duke Math. J. 36 (1969), 559-563.

2. - Density of one graph along another, Proc. Amer. Math. Soc. 20 (1969), 147-150. MR 38 \#1220.

3. J. L. Cornette, Connectivity functions and images on Peano continua, Fund. Math. 58 (1966), 183-192. MR 33 \#6́600.

Auburn University 